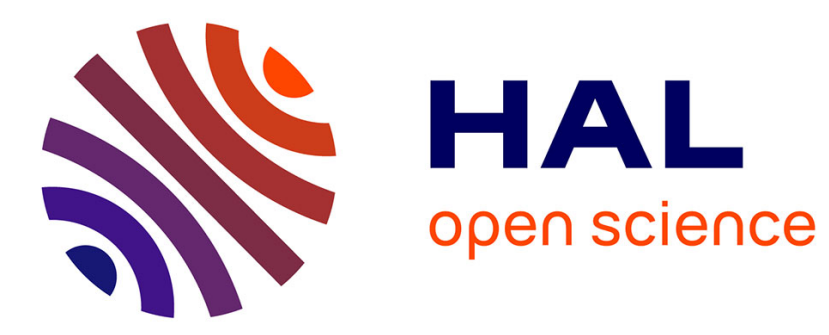

\title{
Study of the Low-Frequency Excess Equivalent Magnetic Noise in GMI-Based Devices
}

Elodie Portalier, Basile Dufay, Christophe P. Dolabdjian, Djamel Seddaoui, Arthur Yelon, David Ménard

\section{> To cite this version:}

Elodie Portalier, Basile Dufay, Christophe P. Dolabdjian, Djamel Seddaoui, Arthur Yelon, et al.. Study of the Low-Frequency Excess Equivalent Magnetic Noise in GMI-Based Devices. IEEE Sensors Journal, 2017, 17 (21), pp.6888 - 6894. 10.1109/JSEN.2017.2748601 . hal-01621526

\section{HAL Id: hal-01621526 https://hal.science/hal-01621526}

Submitted on 13 May 2020

HAL is a multi-disciplinary open access archive for the deposit and dissemination of scientific research documents, whether they are published or not. The documents may come from teaching and research institutions in France or abroad, or from public or private research centers.
L'archive ouverte pluridisciplinaire HAL, est destinée au dépôt et à la diffusion de documents scientifiques de niveau recherche, publiés ou non, émanant des établissements d'enseignement et de recherche français ou étrangers, des laboratoires publics ou privés. 


\title{
Study of the low-frequency excess equivalent magnetic noise in GMI-based devices
}

\author{
E. Portalier*, B. Dufay*, C. Dolabdjian*, D. Seddaoui ${ }^{\dagger}$, A. Yelon ${ }^{\dagger}$, D. Ménard ${ }^{\dagger}$, \\ ${ }^{*}$ Groupe de recherche en Informatique, Image, Automatique et Instrumentation de Caen \\ Normandie Univ, UNICAEN, ENSICAEN, CNRS, GREYC, 14000 Caen, France \\ ${ }_{\dagger}^{\dagger}$ Polytechnique Montréal, Département de génie physique \& Regroupement québécois des matériaux de pointe, \\ Montréal, Québec, Canada H3C3A7
}

\begin{abstract}
Low frequency equivalent magnetic noise levels of four different GMI samples, exhibiting $1 / f$ spectral behavior, have been experimentally determined, for several excitation conditions (involving both dc current and ac excitation amplitude). Under appropriate conditions, for which the noise level at $1 \mathrm{~Hz}$ is minimal, coherence measurements show that the main noise source responsible for this low frequency noise is the intrinsic noise of the GMI sample. The lowest corresponding magnetic noise performances of the four samples were determined. The lowest noise level observed was $17 \mathrm{pT} / \sqrt{\mathrm{Hz}}$ at $1 \mathrm{~Hz}$. It has been proposed that the imaginary part of the magnetic susceptibility, $\chi$ ", a measure of the low frequency magnetization fluctuations, determines the resulting magnetic noise level of the sensor. Its values at $1 \mathrm{~Hz}$ has been evaluated for all four samples. Using these evaluations, expected noise levels at $1 \mathrm{~Hz}$ are computed, based on a previously proposed theoretical model, and compared to the measured noise performances. Under conditions for which correlation measurements shows that system noise is dominated by sensor noise, the two sets of value are in reasonably good agreement, suggesting that the proposed model for low frequency excess noise of GMI materials is essentially correct.
\end{abstract}

Index Terms-Giant magneto-impedance (GMI), Noise, Magnetometer, Magnetization fluctuations.

\section{INTRODUCTION}

The giant magneto-impedance (GMI) effect describes the large variations of the impedance of a soft ferromagnetic conductor submitted to changing external applied magnetic field [1]. Over two decades, it has attracted considerable attention as it appears to be a serious candidate for high sensitivity magnetometry. While low-sensitivity systems have been commercialized for mobile phones since 2002, newer designs exhibiting pico-Tesla resolution are promising for biomedical applications [2]. For high sensitivity magnetometry, an important characteristic is the equivalent magnetic noise level of the sensor since this determines the ultimate detection level.

It has been shown that the noise level in the white noise region, generally above $1 \mathrm{kHz}$, is dominated by electronic conditioning noise, rather than by the intrinsic noise of the GMI sensing element [3], [4]. As a consequence, increasing the intrinsic sensitivity of the sensor until the intrinsic sensor noise dominates the electronic conditioning noise will be advantageous. One method of increasing the intrinsic sensitivity of GMI sensors to the order of $\mathrm{pT} / \sqrt{\mathrm{Hz}}$ at $1 \mathrm{~Hz}$ is the use of a pick-up coil strongly coupled to the GMI element. This is referred to as off-diagonal GMI [5], [6] or orthogonal flux-gate in fundamental mode [7], [8], [9], [10].

With sufficiently high intrinsic sensitivity, equivalent magnetic noise spectral density measurements show that an excess noise level arises at low frequency, exhibiting $1 / f$ behavior [11], while the noise level in the white noise region is still dominated by the electronic conditioning noise. Recently, it has been proposed that this $1 / f$ excess noise is due to low frequency magnetization fluctuations. The theoretical model for classical GMI based on this hypothesis [12] has recently been developed for off-diagonal GMI [13]. The magnetic fluctuations are directly linked to the imaginary part, $\chi$ ", of the magnetic susceptibility. The equivalent magnetic noise level of the sensor is given by

$$
b_{n}^{2}(f)=\frac{\mu_{0}}{\sin ^{2} \theta_{M}} \frac{4 k_{B} T}{2 \pi \Omega} \frac{H_{i n t}^{2}}{M_{S}^{2}} \frac{\chi^{\prime \prime}(f)}{f},
$$

where $M_{S}$ is the absolute value of the magnetization, which makes an angle $\theta_{M}$ with respect to the wire axis (and an angle $\left(\frac{\pi}{2}-\theta_{M}\right)$ with respect to the circumference), which is determined by the effective internal field $H_{\text {int }}$ at static equilibrium. Magnetic anisotropy, including static and dynamic demagnetizing effects, are implicitly included in $H_{\text {int }}$ and $\theta_{M}$. In (1), $\Omega$ is the sample volume, $\mu_{0}$ the vacuum permeability, $k_{B}$ the Boltzmann constant, and $T$ the temperature.

This model has led to plausible results for a particular sensing element [13], [14]. However, a broader comparison is required to demonstrate its general validity. In addition, the effect of the dc current, $I_{d c}$, and of the amplitude of the ac excitation current, $I_{a c}$, on this excess noise, which has previously been discussed in refs. [8], [9], is not fully understood yet. As a consequence, this paper is dedicated to an experimental investigation of the origin of the low frequency excess noise and to a comparison between expected and measured noise performances at $1 \mathrm{~Hz}$ on a set of four GMI samples.

The study of this $1 / f$ excess noise arising at low frequency is relevant only if the noise from the electronic circuitry is low enough so that the intrinsic noise arising from the sensor is the dominant noise source. Consequently, in what follows, we always consider a sensing element consisting of a GMI wire strongly coupled to a pick-up coil as such a configuration enhances the sensitivity compared to a classical GMI sensor. This is one of the conditions required to 
reduce the noise contribution from electronic conditioning circuitry [15]. Nevertheless, it has been shown that the noise arising from magnetization fluctuations affects off-diagonal GMI and classical GMI similarly, if expressed as an equivalent magnetic noise level [12], [13].

This paper is organized as follows. Section II is dedicated to the experimental study of the low frequency excess noise. The arrangements for coherence measurements, which permit the dominant contribution to the total noise level to be determined, are described. The best noise performances obtained for each sample are also presented. Section III concerns the evaluation of $\chi "$ in the linear regime. Section IV is dedicated to the comparison of the measured noise level at $1 \mathrm{~Hz}$ with that expected from the proposed model. We conclude in section $\mathrm{V}$.

\section{LOW FREQUENCY EXCESS NOISE MEASUREMENT}

Previous studies [8], [9] have shown an empirical relation linking the equivalent magnetic noise to both $I_{a c}$ and $I_{d c}$ and have highlighted a proportionality between the noise at $1 \mathrm{~Hz}$ and the inverse of the GMI sensitivity under certain circumstances. Indeed, $I_{a c}$ directly sets the output voltage sensitivity (expressed in $\mathrm{V} / \mathrm{T}$ ) of the device and should be chosen as high as possible so that the effect of the noise contribution from the electronic circuitry is reduced [16]. The dc bias current determines the static equilibrium condition in the GMI wire which plays a role both in the intrinsic sensitivity value, $S_{v}$ (expressed in $\Omega / \mathrm{T}$ ) and in the intrinsic noise level.

\section{A. Experimental conditions}

The setup used for the equivalent magnetic noise measurement is that presented in refs. [11], using a peak detector as the demodulation stage. The static working point, $B_{0}$, is set by positioning a permanent magnet in the proximity of the sensing element, but far enough that the field may be considered to be homogeneous, so as to maximize the intrinsic sensitivity and minimize the average value of the carrier, as discussed in [11].

We studied four different GMI samples based on amorphous $\mathrm{CoFeSiB}$ alloy, from three sources. All the samples are $24 \mathrm{~mm}$ long and were coupled to similar $450 \mu \mathrm{m}$ diameter, 200 turn, pick-up coil. Samples $s_{a c}$ and $s_{m e}$ are as-cast alloys. Sample $s_{a n}$ was the same alloy as $s_{a c}$, annealed so as to reduce its anisotropy dispersion. Sample $s_{g c}$ is glass covered wire sample. Table I summarizes the geometrical, and some magnetic properties of these four samples.

\section{B. Coherence measurements}

First, it is necessary to experimentally qualify the origin of the $1 / f$ excess noise and to find the appropriate excitation conditions which minimize its level. The principle of a coherence spectral measurement lies in the fact that the noise of a single sample is measured through two different noise channels giving the ratio of common noise of the channels to total noise. In other words, it allows one to establish if two signals arise from a common origin or from different one. Considering two real signals $y_{1}(t)$ and $y_{2}(t)$ whose associated power spectral

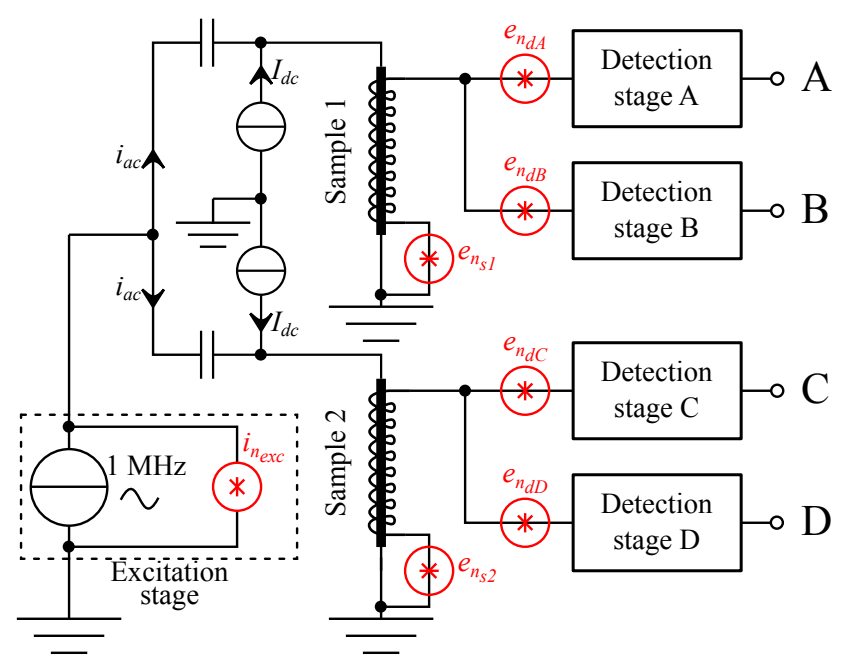

Figure 1: Experimental setup used for cross-correlation measurement. The excitation and detection stage are made of the same electronic circuitry as presented in [11], [15].

densities are $S_{y_{1} y_{1}}(f)$ and $S_{y_{2} y_{2}}(f)$ and whose power interspectral density is $S_{y_{1} y_{2}}(f)$, the coherence spectrum is given by [17], [18]

$$
\gamma_{y_{1} y_{2}}^{2}(f)=\frac{\left|S_{y_{1} y_{2}}(f)\right|^{2}}{S_{y_{1} y_{1}}(f) S_{y_{2} y_{2}}(f)} .
$$

Then, signals $y_{1}$ and $y_{2}$ are considered perfectly correlated (in other words, they possess the same origin) at the frequency, $f$, if $\gamma_{y_{1} y_{2}}(f)=1$. On the contrary, if $\gamma_{y_{1} y_{2}}(f)=0$, they are totally uncorrelated.

Conducting coherence measurements of the noise level on a replicated measuring chain allows one to determine whether the main noise contribution arises from a common part of the setup (correlated noise) or from a separate part (uncorrelated noise). Figure 1 shows a setup which has four outputs: A, B, C and D. For each of these outputs, the ratio between common and separated elements of the measuring chain may be different. Both GMI wires and pickup coils and all four detection stages were as similar as possible. Bias current, $I_{d c}$, is provided separately for the two samples by two different current sources (with high output impedance). Coherence measurements were conducted for all four types of GMI wires presented in Table I.

There are three major noise sources, which are considered to be uncorrelated:

- $i_{n_{\text {exc }}}$ is the noise arising from the excitation stage of power spectral density $S_{n_{\text {exc }}}(f)$,

- $e_{n_{s 1}}$ and $e_{n_{s 2}}$ are the noise due to the equivalent magnetic noise of the samples of power spectral density $S_{n_{s 1}}(f)$ and $S_{n_{s 2}}(f)$,

- $e_{n_{d A}}, e_{n_{d B}}, e_{n_{d C}}$ and $e_{n_{d D}}$ are the noise from the detection stages A, B, C and D, with associated power spectral densities $S_{n_{d A}}(f), S_{n_{d B}}(f), S_{n_{d C}}(f)$ and $S_{n_{d D}}(f)$.

The coherence spectra between outputs $\mathrm{A}$ and $\mathrm{B}$, and between outputs $\mathrm{C}$ and $\mathrm{D}$, which share the same sensing element are described by Eqs. (3) and (4) whereas the coherence spectrum 


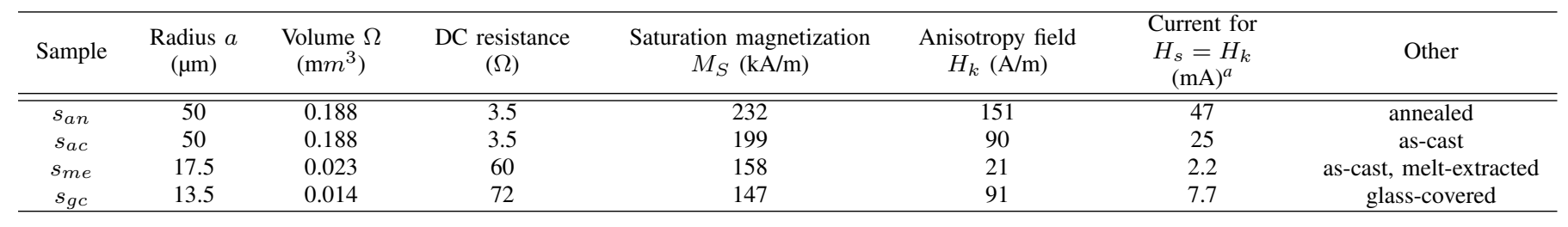

${ }^{a} H_{s}$ is the field at the wire surface.

Table I: Properties of the four samples studied.

between outputs $\mathrm{B}$ and $\mathrm{C}$ (or A and $\mathrm{D}$ ) which only share the excitation stage is described by Eq. (5).

Figure 2 shows an example of a result obtained for output signals delivered by outputs $\mathrm{A}$ and $\mathrm{B}$. The equivalent magnetic noise level, curve (a), is the same for the two outputs and exhibits a $1 / f$ dependence at low frequency along with a white noise floor at higher frequency. The coherence spectrum between both outputs, curve (b), clearly shows that at very low frequency, where the noise spectral density exhibits a $1 / f$ behavior, the correlation is high. This indicates that the main noise arises either from the excitation or from the sensing element. In contrast, in the white noise region ( $f$ higher than $2 \mathrm{kHz}$ ), the correlation decreases indicating that the noise arising from the detection stage becomes the dominant noise source.

Similar measurements were conducted for several combinations of excitation parameters. Figure 3 summarizes the results obtained, showing the equivalent magnetic noise level at $1 \mathrm{~Hz}$ along with the corresponding coherence value $\gamma_{A, B}^{2}$ and $\gamma_{B, C}^{2}$ as a function of $I_{a c}$, for $I_{d c}=10 \mathrm{~mA}$. In this figure, one can distinguish three main regions of $I_{a c}$ :

- At low $I_{a c}\left(\lesssim 8 \mathrm{~mA}_{\text {rms }}\right)$, the low correlation value between outputs $\mathrm{A}$ and $\mathrm{B}$ and outputs $\mathrm{B}$ and $\mathrm{C}$ which shows that the dominant noise is due to non-correlated noise sources (the detection stage in that case). Moreover the equivalent magnetic noise levels at $1 \mathrm{~Hz}$ are roughly proportional to the inverse of the sensitivity. In other words, the corresponding output voltage noise is constant, induced by the conditioning electronic circuitry. This indicates that the dominant noise does not arise from the sensor.

- At high excitation current $\left(I_{a c} \gtrsim 12 \mathrm{~mA}_{\mathrm{rms}}\right)$, the equivalent magnetic noise level increases by a factor 6 over the range studied, while the sensitivity increases by less than a factor 4, indicating that other noise sources are involved compared to the previous case. Now, the correlation value is high between outputs $\mathrm{A}$ and $\mathrm{B}$, that is, the noise level is dominated by a correlated noise source (the sensor's intrinsic noise or the excitation stage noise). The correlation between outputs B and C is still low, indicating that the main noise source is not the excitation stage which is the only common noise source shared between channels B and C. As a consequence, the main noise source is the intrinsic noise of the sensor.

- The intermediate level $\left(8 \mathrm{~mA}_{\text {rms }} \lesssim I_{a c} \lesssim 12 \mathrm{~mA}_{\text {rms }}\right)$ corresponds to the transition between the other two regions and thus exhibit an unequal mix of the previous noise sources.

As a consequence, it appears that if the excitation conditions are favorable, the output noise level at $1 \mathrm{~Hz}$ originates from the sensing element itself.

\section{Effect of $d c$ bias and ac excitation current}

The measurements presented in the previous subsection were completed by investigating the effect of $I_{d c}$ and of $I_{a c}$.

The results are presented in Fig 4. Figure 4a, shows that the minimum noise at $1 \mathrm{~Hz}$ is reached for higher values of $I_{a c}$ as $I_{d c}$ is increased. This is mainly due to the fact that the intrinsic sensitivity (in $\Omega / T$ ) of the sensor decreases with $I_{d c}$ [16]. To compensate for this reduction, it is necessary to increase $I_{a c}$ if the same order of voltage sensitivity value is to be reached. However, this has the advantage that the minimum noise level is slightly lower for high dc bias currents (and high excitation) than that for low de bias. This was previously reported by Butta et al. [8].

Figure $4 \mathrm{~b}$ shows the corresponding coherence value obtained between outputs A and B. As discussed above, it appears that the main noise source is the intrinsic noise of the sensor as soon as the minimum noise level is reached (or exceeded). This latter is obtained if the excitation amplitude, balanced by $I_{d c}$, is high enough.

\section{Magnetic noise performances of four GMI samples}

Finally, the same experimental protocol was conducted for the four GMI samples described in table I. The equivalent magnetic noise at $1 \mathrm{~Hz}$ of the four samples behaves the same as that of sample $s_{a n}$ as described in section II-C. That is, the same conclusions regarding coherence measurements apply and have been verified.

To summarize, Table II gives the measured equivalent magnetic noise level at $1 \mathrm{~Hz}$ of the four samples as a function of dc bias current. As discussed above, the origin of the excess noise at $1 \mathrm{~Hz}$ may be ascribed to an intrinsic noise source only if the correlation value is high enough so that the noise no longer arises from the electronic circuitry of the detection bloc. As a consequence, noise level values are given in the table II only if a correlation value of 0.8 or higher is observed.

\section{EVALUATION OF THE IMAGINARY PART OF THE MAGNETIC SUSCEPTIBILITY}

As discussed in Section I, it has been proposed that the intrinsic noise of the GMI material is due to the low frequency 


$$
\begin{aligned}
& \gamma_{A, B}^{2}(f)=\frac{\left|S_{n_{e x c}}(f)+S_{n_{s 1}}(f)\right|^{2}}{\left(S_{n_{e x c}}(f)+S_{n_{s 1}}(f)+S_{n_{d A}}(f)\right)\left(S_{n_{e x c}}(f)+S_{n_{s 1}}(f)+S_{n_{d B}}(f)\right)} \\
& \gamma_{C, D}^{2}(f)=\frac{\left|S_{n_{e x c}}(f)+S_{n_{s 2}}(f)\right|^{2}}{\left(S_{n_{e x c}}(f)+S_{n_{s 2}}(f)+S_{n_{d C}}(f)\right)\left(S_{n_{e x c}}(f)+S_{n_{s 2}}(f)+S_{n_{d D}}(f)\right)} \\
& \gamma_{B, C}^{2}(f)=\frac{\left|S_{n_{e x c}}(f)\right|^{2}}{\left(S_{n_{e x c}}(f)+S_{n_{s 1}}(f)+S_{n_{d B}}(f)\right)\left(S_{n_{e x c}}(f)+S_{n_{s 2}}(f)+S_{n_{d C}}(f)\right)}
\end{aligned}
$$

\begin{tabular}{|c|c|c|c|c|c|c|c|c|c|c|c|c|}
\hline \multirow{2}{*}{$\begin{array}{c}\text { Sample } \\
I_{d c} \\
(\mathrm{~mA})\end{array}$} & \multicolumn{2}{|c|}{$s_{a n}$} & \multirow[b]{2}{*}{$\begin{array}{c}I_{a c} \\
\left(\mathrm{~mA}_{\mathrm{rms}}\right) \\
\end{array}$} & \multicolumn{3}{|c|}{$s_{a c}$} & \multicolumn{3}{|c|}{$s_{m e}$} & \multicolumn{3}{|c|}{$s_{g c}$} \\
\hline & $\begin{array}{c}\text { noise at } 1 \mathrm{~Hz} \\
(\mathrm{pT} / \sqrt{\mathrm{Hz}})\end{array}$ & $\begin{array}{c}S_{v} \\
(\mathrm{MV} / \mathrm{T})\end{array}$ & & $\begin{array}{c}\text { noise at } 1 \mathrm{~Hz} \\
(\mathrm{pT} / \sqrt{\mathrm{Hz}})\end{array}$ & $\begin{array}{c}S_{v} \\
(\mathrm{MV} / \mathrm{T})\end{array}$ & $\begin{array}{c}I_{a c} \\
\left(\mathrm{~mA}_{\mathrm{rms}}\right)\end{array}$ & $\begin{array}{c}\text { noise at } 1 \mathrm{~Hz} \\
(\mathrm{pT} / \sqrt{\mathrm{Hz}})\end{array}$ & $\begin{array}{c}S_{v} \\
(\mathrm{MV} / \mathrm{T})\end{array}$ & $\begin{array}{c}I_{a c} \\
\left(\mathrm{~mA}_{\mathrm{rms}}\right)\end{array}$ & $\begin{array}{c}\text { noise at } 1 \mathrm{~Hz} \\
(\mathrm{pT} / \sqrt{\mathrm{Hz}})\end{array}$ & $\begin{array}{c}S_{v} \\
(\mathrm{MV} / \mathrm{T})\end{array}$ & $\begin{array}{c}I_{a c} \\
\left(\mathrm{~mA}_{\mathrm{rms}}\right)\end{array}$ \\
\hline 0 & 35 & 5.1 & 8 & & & & & & & & & \\
\hline 0.5 & & & & & & & 306 & 1.3 & 3 & & & \\
\hline 1 & 60 & 4.3 & 8 & & & & 173 & 0.9 & 1 & 695 & 0.6 & 3 \\
\hline 2 & & & & 107 & 3.0 & 8 & & & & & & \\
\hline 3 & & & & 100 & 7.1 & 10 & 55 & 3.5 & 3 & 188 & 1.2 & 5 \\
\hline 5 & 42 & 6.5 & 12 & 37 & 3.4 & 8 & 61 & 2.8 & 3 & 126 & 0.9 & 5 \\
\hline 7 & & & & & & & 43 & 3.4 & 6 & & & \\
\hline 10 & 26 & 5.2 & 12 & 32 & 4.3 & 10 & 46 & 2.8 & 9 & 110 & 1.5 & 7 \\
\hline 15 & 28 & 6.4 & 16 & & & & & & & & & \\
\hline 20 & 22 & 5.6 & 20 & 25 & 1.8 & 12 & & & & & & \\
\hline 40 & 22 & 8.8 & 32 & 17 & 8.2 & 24 & & & & & & \\
\hline
\end{tabular}

Table II: Measured equivalent magnetic noise levels at $1 \mathrm{~Hz}$ and sensitivity, for the four samples studied, for various dc bias currents, and for which the noise level may be ascribed to an intrinsic noise source (correlation degree higher than 0.8). For each sample, the third column indicates the amplitude, $I_{a c}$, of the excitation current, used to obtain this noise level. Best performances of each sample are highlighted in bold characters.

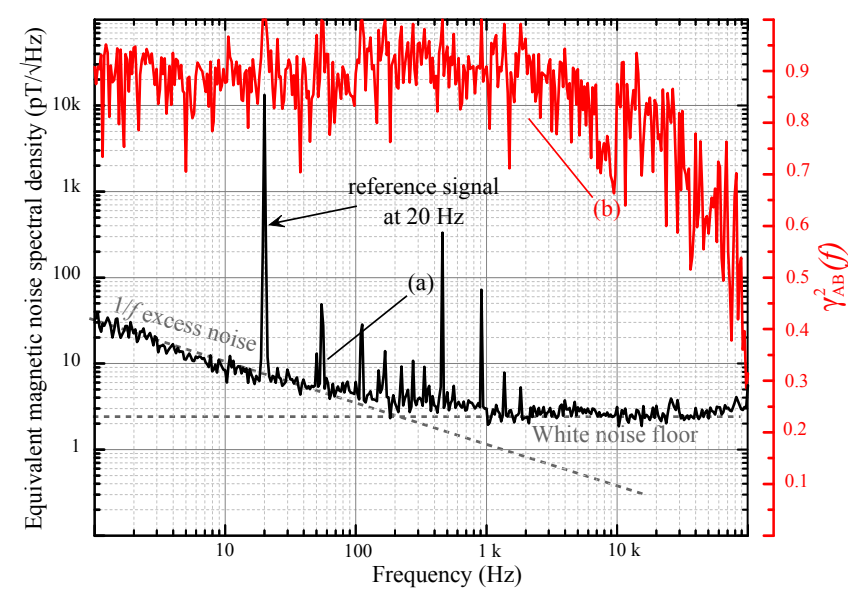

Figure 2: Measured equivalent magnetic noise spectral density (a) and correlation spectrum (b) between outputs A and B, $\gamma_{A, B}^{2}(f)$, for the sample $s_{a n}$. The excitation current is a $1 \mathrm{MHz}$ sine wave of $I_{a c}=16 \mathrm{~m} A_{r m s}$, and $I_{d c}=10 \mathrm{~mA}$. Bandwith of our measurement chain is $100 \mathrm{kHz}$, which corresponds to the max frequency of the spectrum illustrated.

fluctuations of the magnetization direction with a corresponding magnetic noise level given by (1). In this equation, the noise level is directly linked to the static equilibrium state of the sample and to the imaginary part of the magnetic susceptibility, $\chi$ ". To predict the expected noise performances at low frequency, it is necessary to evaluate this last parameter.

This was done for all samples, using the measurement method based on the determination of the real part of the low

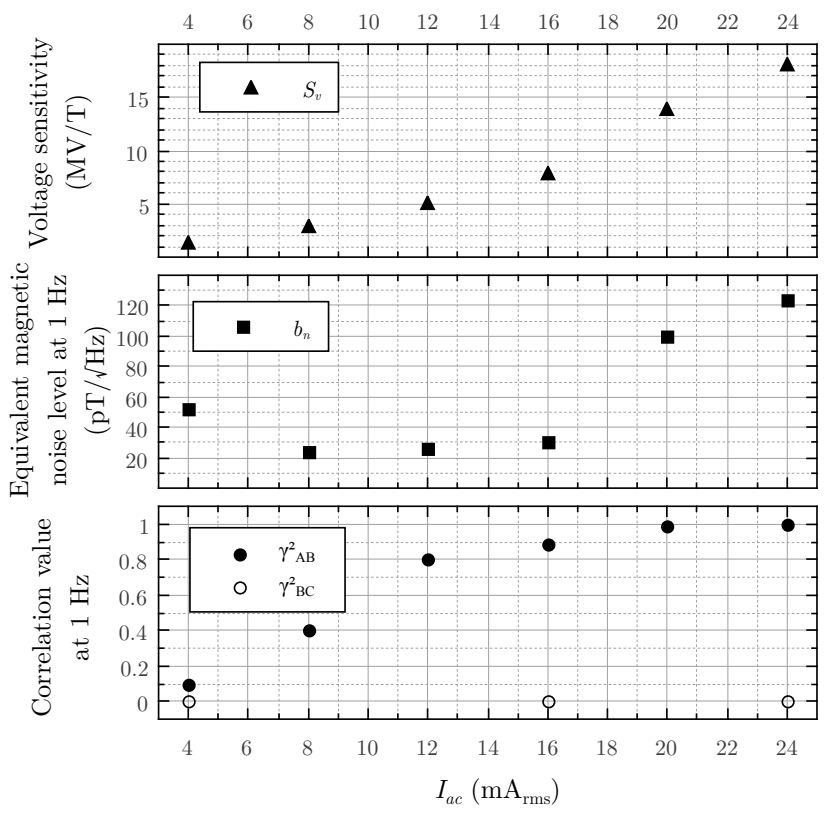

Figure 3: Behavior of the equivalent magnetic noise level, of the voltage sensitivity, in $(\mathrm{V} / \mathrm{T})$, and of the coherence level, at $f=1 \mathrm{~Hz}$, for sample $s_{a n}$, as a function of the excitation current amplitude. The frequency of the excitation current is $1 \mathrm{MHz}$ and $I_{d c}=10 \mathrm{~mA}$. 


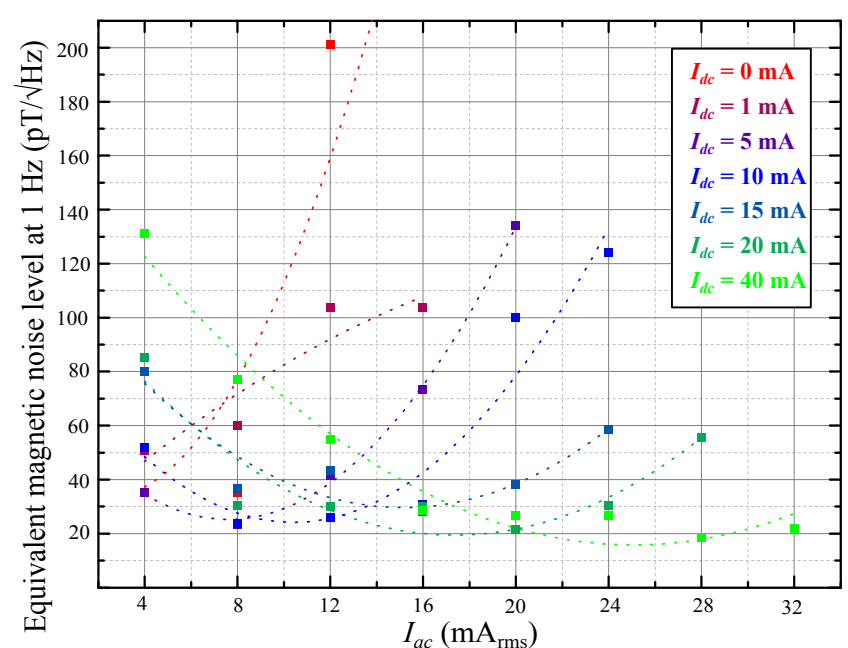

(a)

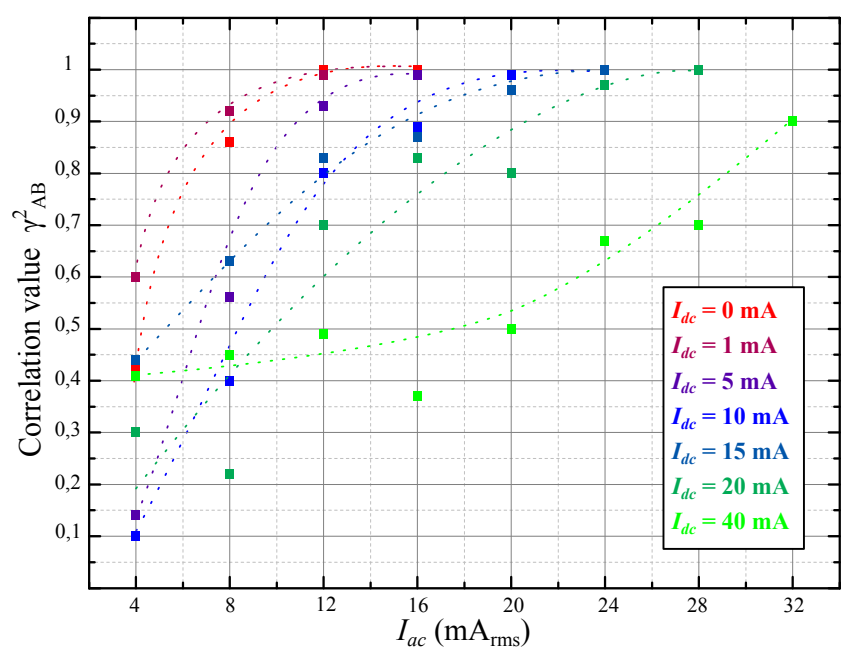

(b)

Figure 4: (a) Equivalent magnetic noise level at $1 \mathrm{~Hz}$ as a function of $I_{a c}$, for several values of $I_{d c}$, and (b) corresponding coherence value between outputs $\mathrm{A}$ and $\mathrm{B}$, for sample $s_{a n}$. Dashed lines are a guide for the eyes.

frequency, linear, impedance of the sample, $\Re(Z)$, as discussed in [14]. The results at the frequency of interest, $f=1 \mathrm{~Hz}$, are presented in Fig. 5. They permit the evaluation of the expected noise at $1 \mathrm{~Hz}$ using Eq. (1).

\section{COMPARISON WITH THE NOISE MODEL}

The equivalent magnetic noise $b_{n}$, in $\mathrm{T} / \sqrt{\mathrm{Hz}}$, is among the most important figures, perhaps the most, to qualify the performance of a magnetometer. In circumstances where the magnetometer is limited by a white noise spectrum due to the electronic circuitry, the equivalent magnetic noise is obtained by dividing the voltage noise, in $\mathrm{V} / \sqrt{\mathrm{Hz}}$, by the sensitivity, in $\mathrm{V} / \mathrm{T}$. It decreases as the sensitivity increases. This is exemplified by the low $I_{a c}$ regime in Figure 3 , as increasing $I_{a c}$ allows us to increase the sensitivity and thus decrease $b_{n}$, up to a certain threshold, corresponding to the gradual transition to what we call the high excitation current regime. Increasing $I_{d c}$ results in higher magnetic stiffness and

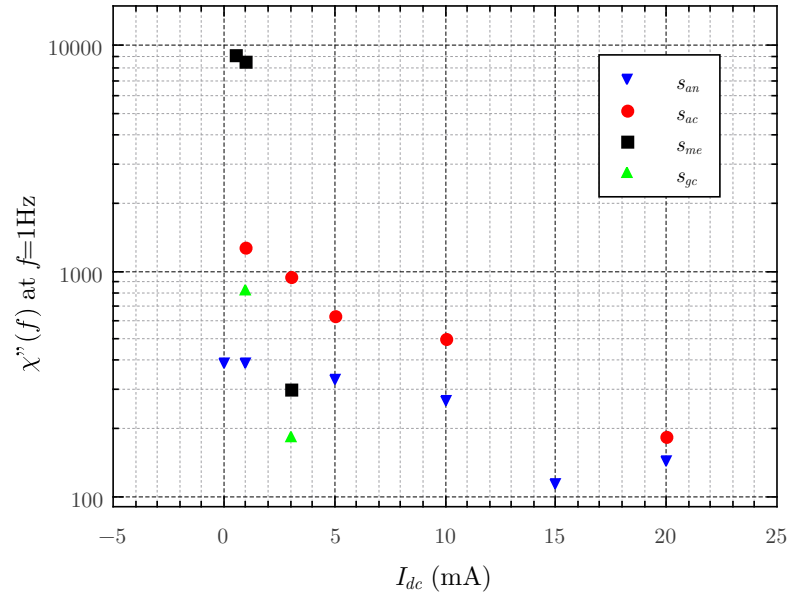

Figure 5: The imaginary part of the magnetic susceptibility, $\chi^{\prime \prime}(f)$, at $f=1 \mathrm{~Hz}$, as a function of the DC bias current. Their evaluation relies on the measurement of $\Re(Z)$ [14]

thus lower sensitivity, requiring correspondingly higher $I_{a c}$ to reach the transition, for which $b_{n}$ reach its lowest values. This shifting of the transition region to higher $I_{a c}$ as $I_{d c}$ increases is well demonstrated in Figure 4a.

We have proposed, on several occasions in the past, that beyond the transition region, the equivalent magnetic noise is limited by intrinsic thermal magnetization fluctuations. The same physical mechanisms responsible for making the sensor very sensitive to an external magnetic field also make it more susceptible to magnetic fluctuations. As a result, beyond a certain point, further increasing the sensitivity does not lead to any improvement in $b_{n}$. In fact, the signal deteriorates in the high $I_{a c}$ regime, as shown by our data and that of previous workers [8], [9]. What we have shown here, using crosscorrelation measurements, is that the most favorable conditions do indeed occur when the noise sources intrinsic to the sensing wires start to dominate the noise spectrum. To identify this intrinsic noise to that caused by magnetization fluctuations, we have estimated $b_{n}$ using Eq. (1) and compared it with intrinsic magnetic noise levels measured at $1 \mathrm{~Hz}$. Notice that an anisotropy field angle, $\theta_{k}$, of $85^{\circ}$ was used in the evaluation of $H_{\text {int }}$.

The result of this comparison is shown in Fig. 6 where the expected noise level at $1 \mathrm{~Hz}$ is plotted versus the measured value for all the samples studied and for several dc bias currents. The red line represents a perfect match between expected and measured values. The data points above this line suggest that the model slightly overestimates the real noise performances. Nevertheless, the proximity with this line shows relatively good agreement, supporting the proposed model.

The optimal noise figures are obtained when $I_{a c}$ is roughly on the order of $I_{d c}$, with slightly improved noise performance as $I_{a c}$ and $I_{d c}$ get higher, but at the cost of power consumption. It is expected that at even higher $I_{a c}-I_{d c}$ combinations, the signal (and perhaps the wire as well) will eventually start to deteriorate due to ever stronger heat dissipation into the metallic wire, but we do not reach that limit with our current setup. 

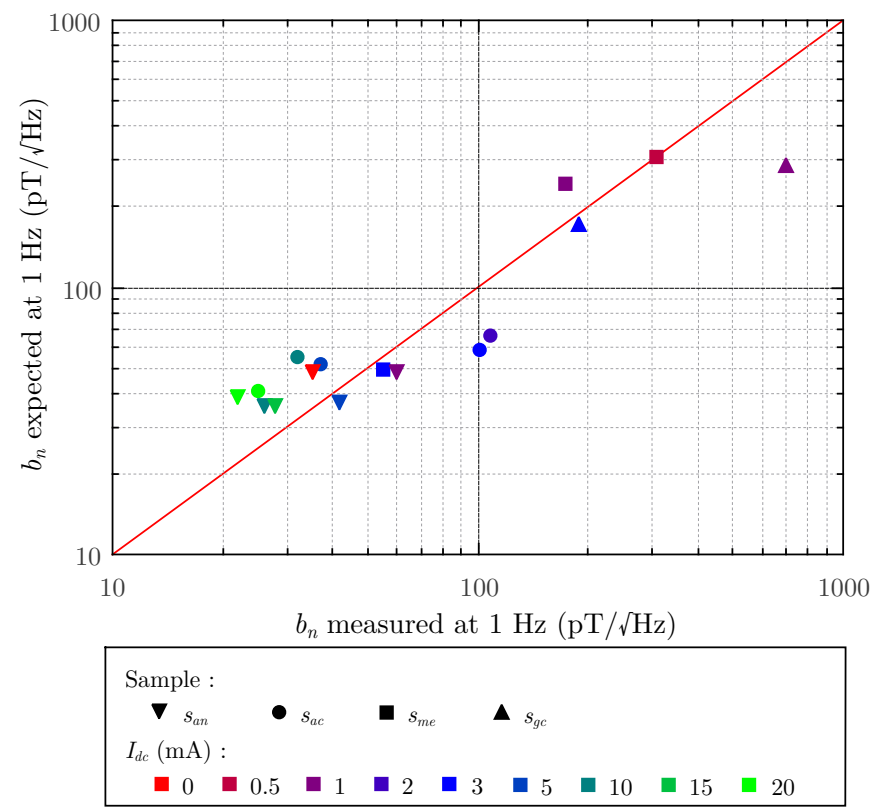

Figure 6: Expected equivalent magnetic noise level at $1 \mathrm{~Hz}$ from (1) versus the measured value, for the four samples studied, and for several dc bias currents. The red line represents a perfect match between expected and measured values.

\section{CONClusion}

Using coherence measurements, it has been shown that under optimal excitation conditions (related to the amplitudes of both ac and dc currents), for which the noise level at $1 \mathrm{~Hz}$ is minimal, the intrinsic magnetic noise of the sensing element is always the dominant noise source, for all samples studied.

The imaginary part of the magnetic susceptibility, $\chi$ ", was measured at low frequency as it plays an important role in the proposed noise model. It is clear that $\chi "(f)$ shows a frequency independent behavior at low frequency, as required by the model to describe $1 / f$ noise.

Finally, the comparison between the expected noise levels at $1 \mathrm{~Hz}$ and those measured yields good agreement, suggesting that the proposed model for estimating the low frequency excess noise of GMI materials is correct. This may help in the search for material improvements in low-frequency equivalent magnetic noise of GMI-based magnetometers.

\section{AKNOWLEDGMENTS}

We are grateful to Dr. M. Butta of the Czech Technical University, Prague, and to Professor H. Chiriac, of the National Institute of Research and Development for Technical Physics, Iasi, both of whom provided samples and characterization information for this study.

\section{REFERENCES}

[1] M. Knobel, M. Vázquez, and L. Kraus, "Giant Magnetoimpedance," in Handbook of magnetic materials, Buschow, Ed. Germany: Elsevier, 2003 , vol. 15 , pp. $497-563$
[2] T. Uchiyama, K. Mohri, Y. Honkura, and L. V. Panina, "Recent advances of pico-Tesla resolution magneto-impedance sensor based on amorphous wire CMOS IC MI sensor," IEEE Transactions on Magnetics, vol. 48, no. 11 , pp. 3833-3839, november 2012.

[3] L. Ding, S. Saez, and C. Dolabdjian, "Low Frequency Giant Magnetoimpedance Magnetometer Noise Versus Electronic Conditioning," Sensor Letters, vol. 5, no. 1, pp. 248-251, Mar. 2007.

[4] L. G. C. Melo, D. Ménard, A. Yelon, L. Ding, S. Saez, and C. Dolabdjian, "Optimization of the magnetic noise and sensitivity of giant magnetoimpedance sensors," Journal of Applied Physics, vol. 103, no. 3, p. $033903,2008$.

[5] S. Sandacci, D. Makhnovskiy, L. Panina, K. Mohri, and Y. Honkura, "Off-Diagonal Impedance in Amorphous Wires and Its Application to Linear Magnetic Sensors," IEEE Transactions on Magnetics, vol. 40, no. 6, pp. 3505-3511, Nov. 2004.

[6] M. Malatek, B. Dufay, S. Saez, and C. Dolabdjian, "Improvement of the off-diagonal magnetoimpedance sensor white noise," Sensors and Actuators A: Physical, vol. 204, pp. 20-24, Dec. 2013.

[7] E. Paperno, "Suppression of magnetic noise in the fundamental-mode orthogonal fluxgate," Sensors and Actuators A: Physical, vol. 116, no. 3, pp. 405-409, Oct. 2004.

[8] M. Butta, S. Yamashita, and I. Sasada, "Reduction of Noise in Fundamental Mode Orthogonal Fluxgates by Optimization of Excitation Current," IEEE Transactions on Magnetics, vol. 47, no. 10, pp. 3748 3751, Oct. 2011.

[9] M. Butta and I. Sasada, "Sources of Noise in a Magnetometer Based on Orthogonal Fluxgate Operated in Fundamental Mode," IEEE Transactions on Magnetics, vol. 48, no. 4, pp. 1508-1511, Apr. 2012.

[10] R. Bazinet, A. Jacas, G. A. B. Confalonieri, and M. Vazquez, "A low-noise fundamental-mode orthogonal fluxgate magnetometer," IEEE Transactions on Magnetics, vol. 50, no. 5, pp. 2013-2015, 2014.

[11] B. Dufay, S. Saez, C. Dolabdjian, A. Yelon, and D. Ménard, "Characterization of an Optimized Off-Diagonal GMI-Based Magnetometer," IEEE Sensors Journal, vol. 13, no. 1, pp. 379-388, Jan. 2013.

[12] C. Dolabdjian, B. Dufay, S. Saez, A. Yelon, and D. Ménard, "Is Low Frequency Excess Noise of GMI Induced by Magnetization Fluctuations?" Key Engineering Materials, vol. 605, pp. 437-440, Apr. 2014.

[13] B. Dufay, E. Portalier, S. Saez, C. Dolabdjian, A. Yelon, and D. Ménard, "Low frequency excess noise source investigation of off-diagonal GMIbased magnetometers," IEEE Transactions on Magnetics, vol. 53, no. 1, pp. 400106 1-6, Jan. 2017

[14] E. Portalier, B. Dufay, C. Dolabdjian, D. Seddaoui, D. Ménard, and A. Yelon, "Evaluation of the imaginary part of the magnetic susceptibility, , and application to the estimation of the low frequency, 1/f, excess noise in GMI sensors," IEEE Transactions on Magnetics, in press, DOI: 10.1109/TMAG.2017.2686805.

[15] B. Dufay, S. Saez, C. Dolabdjian, A. Yelon, and D. Ménard, "Impact of Electronic Conditioning on the Noise Performance of a Two-Port Network Giant MagnetoImpedance Magnetometer," IEEE Sensors Journal, vol. 11, no. 6, pp. 1317-1324, Jun. 2011.

[16] L. Ding, S. Saez, C. Dolabdjian, P. Ciureanu, L. G. C. Melo, A. Yelon, and D. Ménard, "Intrinsic Giant Magnetoimpedance Noise Reduction by DC Bias," Sensor Letters, vol. 5, no. 1, pp. 176-179, mar 2007.

[17] J. Briaire and L. K. J. Vandamme, "Uncertainty in Gaussian noise generalized for cross-correlation spectra," J. Appl. Phys, vol. 84, no. 8, pp. 4370-4374, Oct. 1998.

[18] J. Larsen, "Correlation functions and power spectra," Section for Cognitive Systems, Informatics and Mathematical Modelling, Technical University of Denmark, 2009. 